\title{
Geomechanical properties of the Vaca Muerta Formation
}

\author{
D. Nicolas Espinoza ${ }^{1, *}$ \\ ${ }^{1}$ Hildebrand Department of Petroleum and Geosystems Engineering, The University of Texas at Austin, USA
}

\begin{abstract}
Unconventional oil and gas resources have transformed the energy industry in the United States. Likewise, other countries have followed up with hydrocarbon exploitation from tight unconventional formations. One of the most developed unconventional fields outside North America is the Vaca Muerta Formation in the Neuquén Basin, Argentina. This study summarizes experimental results of quasi-static loading and elastic wave propagation in rocks from the Vaca Muerta Formation along the entire producing interval. The work summarizes experimental results from our laboratory and from the literature. The results highlight the variability of mechanical properties with mineralogy and composition, and the mechanical anisotropy induced by layering. The discussion section explores the implications of spatial variability and anisotropy on in-situ stresses and fluid-driven fractures. Accurate characterization of rock mechanical properties is critical for predicting the geometry of fluid-driven fractures in unconventional rock formations, particularly those subjected to a tectonically active environment such as the Vaca Muerta Formation.
\end{abstract}

\section{Introduction}

The energy resources available in unconventional oil and gas are vast in many countries including the United States, Canada, China, Russia, and Argentina [1]. The exploitation of these resources started in the United States (see example in Fig. 1). Other countries followed a few years later. One of the most notable examples is the Vaca Muerta Formation, particularly regarding oil production (Fig. 1). Unlike many unconventional formations in the United States, the Vaca Muerta Formation is subjected to an active tectonic environment with horizontal stresses that approach the magnitude of vertical stress [2-4]. As a result, the variation of geomechanical properties with lithology and depth can switch the stress regime and affect the geometry of fluid-driven fractures.

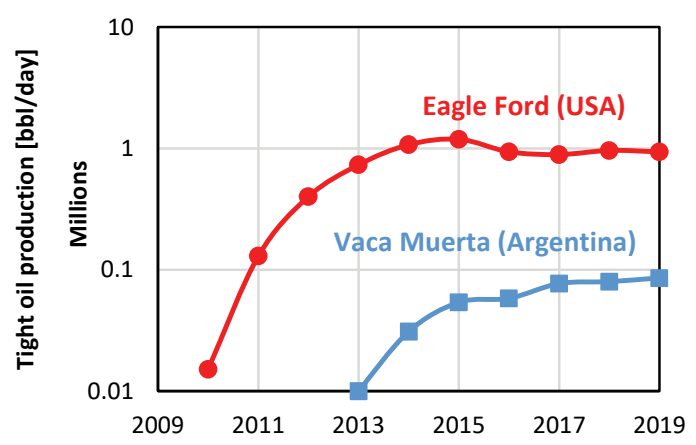

Fig. 1. Comparison of tight oil production between the Vaca Muerta Shale in Argentina and the Eagle Ford Shale in the United States from various sources. The Vaca Muerta Shale is expected to produce at a level as high or higher than many tight oil shales in the United States.

\footnotetext{
* Corresponding author: espinoza@austin.utexas.edu
}

The Vaca Muerta Formation is part of the Neuquén Basin in Argentina [5-6]. The depth varies from 2 to 3.5 $\mathrm{km}$. The total thickness varies from $200 \mathrm{~m}$ to $600 \mathrm{~m}$ increasing in NW direction. The formation is comprised by various rock lithologies formed in a marine environment with majority of mudstones, marls, limestones, and organic shales and minority of igneous sills and veins of fibrous calcite, usually referred to as "beef" [7-8]. The predominant minerals are quartz, calcite and illite clay. The Vaca Muerta Formation is an excellent source rock with total organic content (TOC) that ranges from 2 to $12 \%$. Both TOC and clay content increase as depth increases and remain relatively high at the bottom of the formation $(30-40 \mathrm{~m})$ which is constant throughout the basin [5-6].
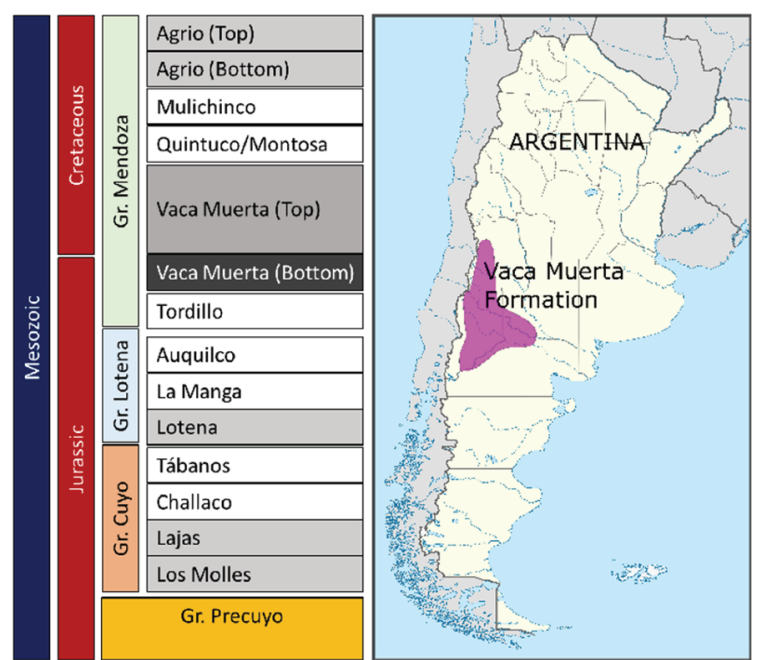

Fig. 2. General stratigraphic column of the Neuquén Basin around the Vaca Muerta Formation and geographical location. 
The objective of this paper is to summarize the results of two geomechanical studies of the Vaca Muerta Formation with emphasis on variation of properties with lithology and mineralogy, and their impact on the in-situ stresses which have a first order influence on the propagation of hydraulic fractures. The reported values combine measurements from well logging and from laboratory experiments.

\section{Bulk Mass Density}

The bulk (or total) mass density measurements are available from laboratory tests and calibrated gamma-ray density well logs. The clay and kerogen volume fractions is available from geochemical well logs. Whenever unavailable, the approximated kerogen volume fraction $[\mathrm{vol} \%]$ is estimated as two-times the total organic content (TOC) [wt\%] [9].

The bulk mass density of rocks in the Vaca Muerta formation vary from $\sim 2.8$ to $2.2 \mathrm{~g} / \mathrm{cm}^{3}$, decreasing in mass density as the volume fraction of clay and kerogen increases (Fig. 3). The highest mass density corresponds to depths with the highest carbonate content. The variability of mineralogy in the Vaca Muerta Formation is quite large. Clay content is usually less than $30 \%$, with carbonate and quartz content that vary from 10 to $80 \%$ [6]. The calcite content is inversely proportional to the clay content [8].

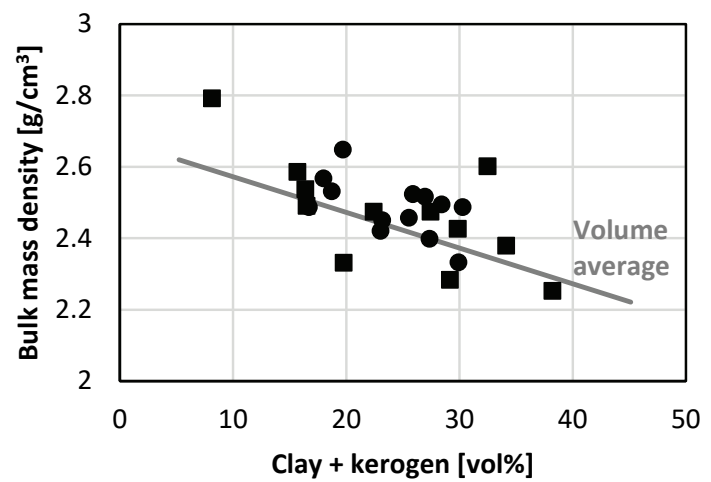

Fig. 3. Bulk mass density of samples from the Vaca Muerta Formation. Mass density decreases with clay and kerogen volume fraction. Clay content positively correlates with kerogen volume fraction. Symbols: (०) Ref [7], (口) Ref. [8]. Line: Eq. (1).

The prediction of bulk mass density $\rho_{\text {bulk }}$ is a straightforward volume average of the constituents.

$$
\rho_{\text {bulk }}=\phi \rho_{f}+f_{1} \rho_{1}+f_{2} \rho_{2}+\ldots+f_{i} \rho_{i}
$$

where the volume fractions are $\phi+f_{1}+f_{2}+\ldots+f_{i}=1$. Fig. 3 shows the volume average predictions assuming the following properties:

- Low TOC rock: $5 \%$ porosity, $0.5 \%$ kerogen, $28.5 \%$ quartz, $61.3 \%$ carbonate, $4.7 \%$ clay.

- Medium TOC rock: 5\% porosity, 10.9\% kerogen, $28.5 \%$ quartz, $41.3 \%$ carbonate, $14.3 \%$ clay.
- High TOC rock: 5\% porosity, 21.4\% kerogen, $28.5 \%$ quartz, $21.4 \%$ carbonate, $23.7 \%$ clay.

The chosen volume fractions correspond to trends interpreted from the values measured by ref. [8]. Other assumptions include: porosity filled with a fluid with $\rho_{f}=$ $1 \mathrm{~g} / \mathrm{cm}^{3}, \rho_{\text {kerogen }}=1.4 \mathrm{~g} / \mathrm{cm}^{3}, \rho_{\text {quartz }}=2.65 \mathrm{~g} / \mathrm{cm}^{3}, \rho_{\text {carbonate }}$ $=2.78 \mathrm{~g} / \mathrm{cm}^{3}$ (calcite and dolomite average), and $\rho_{\text {clay }}=$ $2.2 \mathrm{~g} / \mathrm{cm}^{3}$ taking into account bound water porosity and density.

\section{Small-Strain Stiffness}

Elastic wave propagation yields the dynamic small strain stiffness. This measurement can be performed in the field or in the laboratory. For vertical transverse isotropic media, the P-wave (constrained) modulus along the axis of symmetry is $C_{33}$ and the $\mathrm{P}$-wave modulus perpendicular to the axis of symmetry is $C_{11}$. Sedimentary geological media are characterized by layering. Thus, the modulus parallel to bedding is $C_{11}$, usually in horizontal direction, and the modulus perpendicular to bedding is $C_{33}$, usually in vertical direction.

Laboratory measurements in the Vaca Muerta Formation show that the P-wave modulus of recovered rock samples varies from $\sim 25$ to $80 \mathrm{GPa}$ (equivalent to $\mathrm{P}$ wave velocities from $\sim 3,350$ to $5,600 \mathrm{~m} / \mathrm{s}$ ) with strong trends as a function of clay and kerogen volume fractions, and as a function of orientation with respect to bedding (Fig. 4). Other tight rocks in North America, sources of unconventional oil and gas, exhibit similar trends [10].

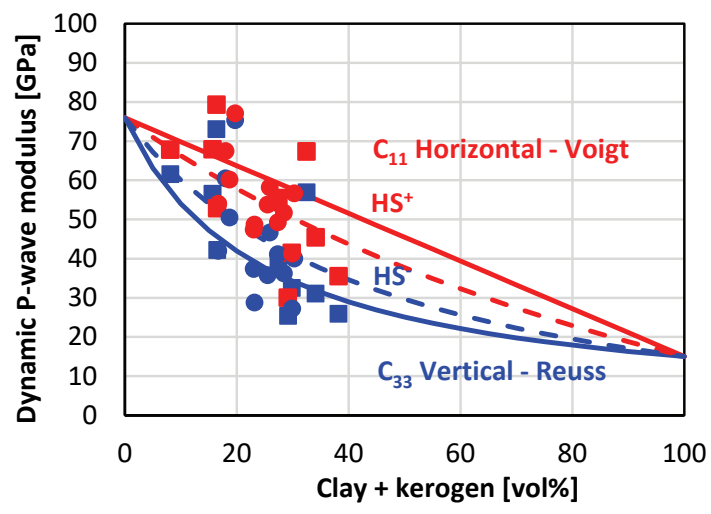

Fig. 4. Dynamic P-wave modulus $C_{11}$ (horizontal) and $C_{33}$ (vertical) of Vaca Muerta rock samples measured in the laboratory decreases with increasing clay and kerogen volume fractions. Symbols: (०) Ref [7], (口) Ref. [8]. The lines correspond to Eqs. (2-3) and Hashin-Shtrikman bounds.

The assumption of perfectly defined horizontal layers permits estimating the bounds for the P-wave modulus of vertical transverse isotropic rocks. The effective P-wave modulus perpendicular to bedding $C_{33}$ can be obtained from assuming that the incremental vertical stress is the same in all layers,

$$
C_{33}=\left(\frac{f_{\text {soft }}}{M_{\text {soft }}}+\frac{f_{\text {stiff }}}{M_{\text {stiff }}}\right)^{-1}
$$


where $f_{\text {soft }}$ and $f_{\text {stiff }}$ are the volume fractions of the soft and stiff layers, and $M_{\text {soft }}$ and $M_{\text {stiff }}$ are the corresponding Pwave (constrained) moduli [11]. This equation assumes isotropic layers and includes porosity within each fraction. An extension to inherently anisotropic layers is available elsewhere [11]. Here, we assume two types of isotropic materials following the simplification proposed by ref. [10], where the soft fraction is composed by clay and organic matter with best-fit constrained modulus $M_{\text {soft }}$ $=15.0 \mathrm{GPa}$ and the stiff fraction is composed by quartz, carbonate, and other minerals with best-fit $M_{\text {stiff }}=75.9$ $\mathrm{GPa}$. The best fit penalizes four times data within bounds to better capture delimiting trends. Similar values are provided elsewhere taking a Hill average of intrinsic values of the mineral phases [10]. Eq. (2) is equivalent to a Reuss-average lower bound.

The effective $\mathrm{P}$-wave modulus parallel to bedding $C_{11}$ for a biphasic layered media (assuming the Poisson ratio $v_{\text {soft }}=v_{\text {stiff }}$ ) can be obtained assuming that the incremental horizontal strain is the same in all layers, such that

$$
C_{11}=f_{\text {soft }} M_{\text {soft }}+f_{\text {stiff }} M_{\text {stiff }} \text {. }
$$

This estimation results in a Voigt-average upper bound. Fig. 4 shows that the proposed theoretical bounds delimit well experimental measurements. The best fit for $M_{\text {soft }}$ and $M_{\text {stiff }}$ also includes predictions from Eq. (3). Values far from bounds indicate that the rock may not be perfectly layered. In addition to Voigt and Reuss averages, Fig. 4 shows Hashin-Shtrikman bounds which assume homogeneous distributions of the stiff and soft phases [11].

\section{Large-Strain Stiffness}

The large-strain stiffness is measured in a triaxial apparatus with quasi-static loading under confined conditions. Deviatoric loading under constant confinement results in direct measurements of Young's modulus and Poisson's ratio. Typical strain magnitudes vary from $10^{-3}$ to $10^{-2}$.

The Young modulus of rock samples from the Vaca Muerta Formation varies from 12 to $50 \mathrm{GPa}$ (Fig. 5). Similar to P-wave stiffness, the Young modulus of Vaca Muerta rock samples has strong dependencies on the volume fraction of clay and kerogen, and on the direction of loading with respect to bedding.

The theoretical bounds for quasi-static Young's modulus can be also predicted with the Voigt and Reuss averages for perfectly layered media. The vertical Young modulus $E_{V}$ and the horizontal Young modulus $E_{H}$ are

$$
\begin{gathered}
E_{V}=\left(\frac{f_{\text {soft }}}{E_{\text {soft }}}+\frac{f_{\text {stiff }}}{E_{\text {stiff }}}\right)^{-1} \\
E_{H}=f_{\text {soft }} E_{\text {soft }}+f_{\text {stiff }} E_{\text {stiff }} .
\end{gathered}
$$

The best fit of Eq. (4) and (5) to the experimental data yields $E_{\text {stiff }}=46.7 \mathrm{GPa}$ and $E_{\text {soft }}=6.3 \mathrm{GPa}$. These results combined with the best fit of P-wave modulus suggest a ratio between static and dynamic Young moduli of $\sim 0.70$.

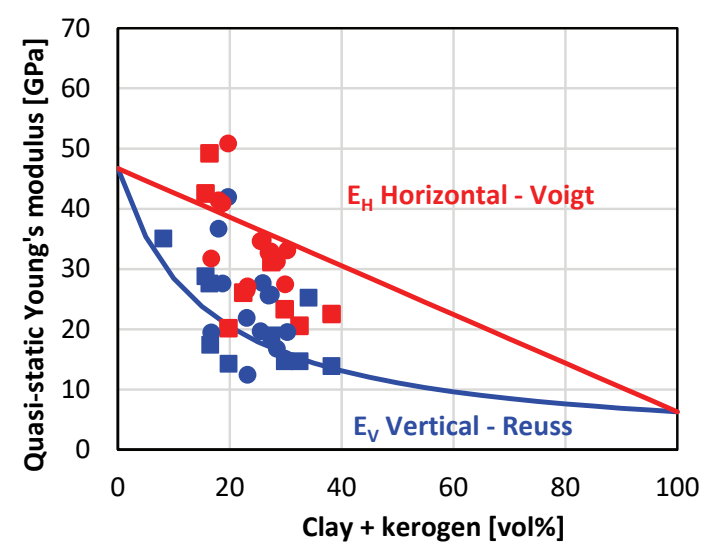

Fig. 5. Quasi-static Young modulus of samples from the Vaca Muerta Formation measured in the laboratory, and bounds for a perfectly layered medium (Eqs. 4-5). Symbols: (०) Ref [7], ( $\square$ ) Ref. [8].

\section{Discussion}

\subsection{Mechanical anisotropy}

Laboratory and field results show that rocks from the Vaca Muerta Formation are anisotropic, with anisotropy increasing as the volume fractions of clay and kerogen increase (Fig. 6). Similar observations are valid for other tight rocks in North America [10]. Other studies rather use the content of carbonate mineral as a controlling agent for stiffness and anisotropy [4,12]. Both approaches yield similar results.

The P-wave modulus anisotropy is usually quantified through the Thomsen parameter $\varepsilon$ [13],

$$
\varepsilon=\frac{C_{11}-C_{33}}{2 C_{33}} \text {. }
$$

Fig. 6a shows that the experimental measurements of P-wave modulus anisotropy are mostly lower than the theoretical prediction of an ideal layered medium with the best fitted stiffness values for the stiff and soft fractions. The maximum anisotropy occurs for equal volume fraction of the soft and stiff phases.

Likewise, the quasi-static stiffness can be quantified by the ratio between the horizontal and vertical Young moduli $E_{h} / E_{v}$. The experimental data for quasi-static stiffness show higher anisotropy than the experimental data for P-wave modulus (Fig. 6a). Plastic and viscous strains, non-negligible in quasi-static loading tests, might be responsible for such phenomenon. Recent studies show that high volume fraction of clays and kerogen increase visco-elastic deformation $[14,15]$. 
(a)

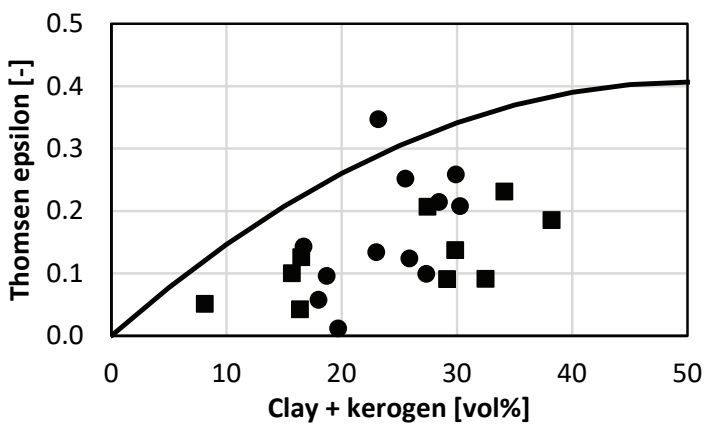

(b)

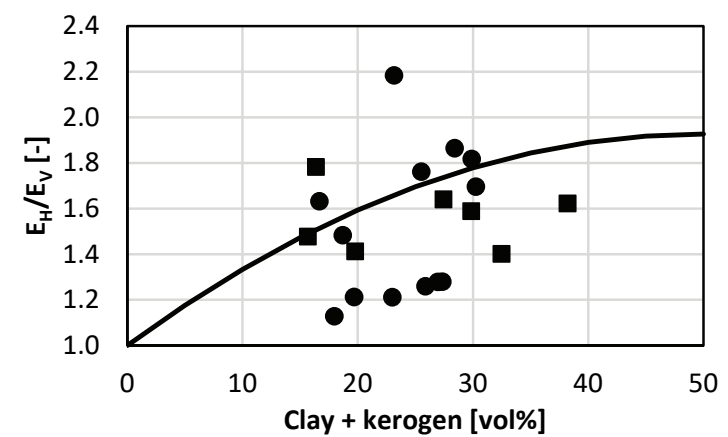

Fig. 6. Mechanical anisotropy of Vaca Muerta rock samples. (a) P-wave stiffness anisotropy. (b) Quasi-static Young modulus anisotropy. The quasi-static stiffness anisotropy is closer to the theoretical prediction than the P-wave modulus anisotropy. Symbols: (०) Ref [7], (口) Ref. [8]. Lines from Eqs. (2-6).

\subsection{Mechanical Stratigraphy}

The variation of mechanical properties with depth has a first order impact on the variation of the minimum principal total stress $S_{3}$, both in magnitude and direction. For example, an anisotropic poro-elastic model predicts the following total minimum horizontal total stress $S_{h}$ as a function of pore pressure $P_{p}$, overburden $S_{V}$, mechanical properties and tectonic strains $\varepsilon_{h}$ (minimum) and $\varepsilon_{H}$ (maximum),

$$
\begin{gathered}
S_{h}=\alpha_{H} P_{p}+\frac{v_{V}}{1-v_{H}} \frac{E_{H}}{E_{V}}\left(S_{V}-\alpha_{V} P_{p}\right)+ \\
\frac{E_{H}}{1-v_{H}^{2}}\left(\varepsilon_{h}+v_{H} \varepsilon_{H}\right)
\end{gathered}
$$

where $\alpha$ is the Biot coefficient and $v$ is the Poisson ratio, with their corresponding directionalities: horizontal $(\mathrm{H})$ and vertical (V). The second term on the right-hand side of the equation captures stress changes solely caused by rock anisotropy. The third term captures the stress changes imposed by horizontal tectonic strains, that have a large contribution in active tectonic environments, such as in the Vaca Muerta Formation. Additional changes of stress can be caused by visco-elastic stress relaxation, particularly in clay and kerogen rich rocks [14].

Fig. 7 shows an example of variability of the quasistatic horizontal Young modulus as a function of depth. The elastic modulus is calculated from the best fit of the data in Fig. 5 with a Voigt average (Eq. 5). The results highlight the variability of the horizontal stiffness. For example, the layers in the vicinity of depths $15 \mathrm{~m}$ from bottom and $37 \mathrm{~m}$ from bottom would build up horizontal stress in the presence of tectonic strains and act as barriers for propagation of vertical fluid-driven fractures. These two depths are characterized by low volume fraction of clay and kerogen. An alternative and more accurate approach consists in correlating all the anisotropic stiffness coefficients $C_{i j}$ to predict mechanical stratigraphy and anisotropy $[3,8]$. The method employed in this paper highlights the influence of mineralogy through a direct relationship between stiffness and mineralogy. (a)

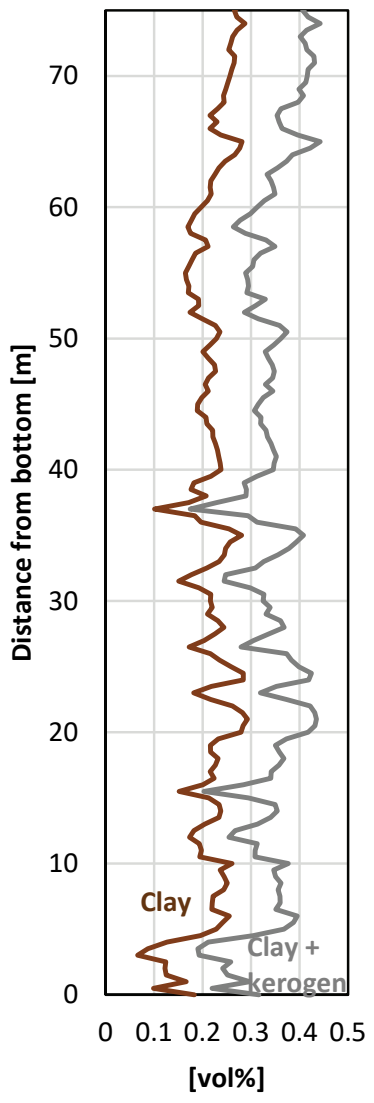

(b)

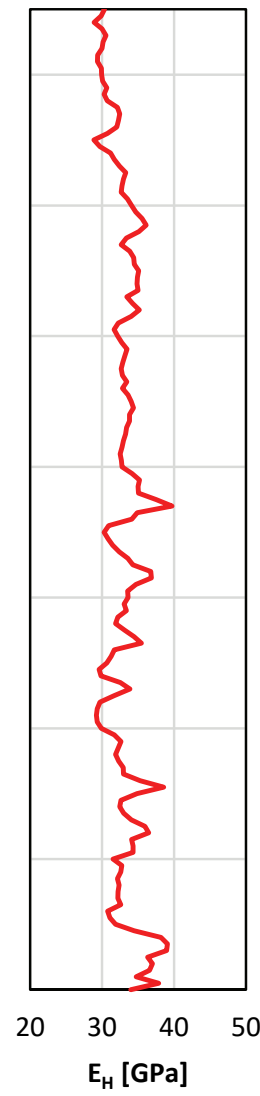

Fig. 7. Example of mechanical stratigraphy close the base of the Vaca Muerta Formation near El Trapial field illustrated by the variation of the horizontal Young modulus. Original clay and organic matter fraction from ref [8]. The value of $E_{H}$ is a Voigtaverage estimation from Fig. 5.

\section{Conclusion}

The Vaca Muerta Formation exhibits marked heterogeneity in mechanical properties caused by variations in lithology and rock facies. The heterogeneity can be partially explained by the presence of relatively soft fractions of clay and kerogen, which align preferentially in horizontal direction and cause rock anisotropy. As a result, the horizontal stiffness is larger than the vertical stiffness. 
The assumption of a perfectly layered model permits finding the stiffness of the soft and stiff fractions and predict bounds for the vertical and horizontal stiffness of the rocks as a function of soft and stiff volume fractions. The combination of Voigt and Reuss bounds captures the experimentally measured mechanical anisotropy but it usually overestimates it. The mechanical anisotropy is larger in quasi-static stiffness than in P-wave stiffness, likely as a result of plastic and creep strains.

Mechanical stratigraphy has a first order impact on in-situ stresses and propagation of fluid-driven fractures. Rock layers with low clay and kerogen are potential fracture barriers for the growth of vertical fractures in shales within tectonically active environments, such as the Vaca Muerta Formation. Optimizing landing points and fracture geometry are essential for economic completion and production shale reservoirs.

\section{References}

1. EIA. Technically Recoverable Shale Oil and Shale Gas Resources: An Assessment of 137 Shale Formations in 41 Countries Outside the United States. U.S. Energy Information Administration, (2013)

2. Guzmán, C., Cristallini, E., Bottesi, G. Tectonics, 26(3), (2007)

3. Frydman, M., Pacheco, F., Pastor, J., Canesin, F. C., Caniggia, J., Davey, H. SPE-180965-MS. In SPE Argentina Exploration and Production of Unconventional Resources Symposium. Society of Petroleum Engineers, (2016)

4. Cuervo, S., Adachi, J., Lombardo, E. ARMA 180856, In 52nd US Rock Mechanics/Geomechanics Symposium, American Rock Mechanics Association, (2018)

5. Chebli, G., Mendiberri, H., Giusiano, A., Ibáñez, G., Alonso, J., Stinco, L. P. In Trabajos Técnicos: VIII Congreso de Exploración y Desarrollo de Hidrocarburos (pp. 669-692), (2011)

6. Askenazi, A., Biscayart, P., Cáneva, M., Montenegro, S., Moreno, M. In SPE Young Professional Committee, 1-20, (2013)

7. Varela, R. A., Marchal, D., Perez Mazas, A. M., Porras, J., Sattler, F., Cavazzoli, G., Lagarrigue, E. ARMA 16-095, In 50th US Rock Mechanics/Geomechanics Symposium, Houston, Texas, (2016).

8. Sosa Massaro, A., Espinoza, D. N., Frydman, M., Barredo, S., Cuervo, S. Journal of South American Earth Sciences, 79, 472-488, (2017)

9. Passey Q. R., Bohacs K. M., Esch W. L., Klimentidis R., Sinha S. In (2010). SPE 131350, (2010)

10. Sone, H., Zoback, M. D. Geophysics, 78(5), D381D392, (2013)

11. Mavko, G., Mukerji, T., Dvorkin, J. The rock physics handbook. (Cambridge university press, 2020)
12. Sosa Massaro, A., Espinoza D. N., Frydman M., Benitez P. E., Speranza T. In $10^{\circ}$ Congreso de Exploración y Desarrollo de Hidrocarburos. IAPG (2018)

13. Thomsen, L. Weak elastic anisotropy. Geophysics, 51(10), 1954-1966, (1986)

14. Sone, H., \& Zoback, M. D. International Journal of Rock Mechanics and Mining Sciences, 69, 120-132, (2014)

15. Hasbani, J. H., \& Hryb, D. E. ARMA 18-1214, In 52nd US Rock Mechanics/Geomechanics Symposium, American Rock Mechanics Association, (2018) 